\title{
ELEMENTARY CONSIDERATIONS FOR CLASSES OF MEROMORPHIC UNIVALENT FUNCTIONS
}

\author{
SAMINATHAN PONNUSAMY AND KARL-JOACHIM WIRTHS
}

\begin{abstract}
In this article we consider functions $f$ meromorphic in the unit disk. We give an elementary proof for a condition that is sufficient for the univalence of such functions. This condition simplifies and generalizes known conditions. We present some typical problems of geometrical function theory and give elementary solutions in the case of the above functions.
\end{abstract}

\section{IntRoduction AND MAin Results}

Let $\mathbb{D}=\{z:|z|<1\}$ and $f$ be meromorphic in $\mathbb{D}$. Much research in the last century has been done concerning such functions that are injective or univalent. Many conditions that are sufficient for univalence of analytical or geometrical character have been found and considered in detail. The present paper is devoted to the following condition of such type that has been found by Aksentév in 1958, see [1], and proved again with another method by Nunokawa and Ozaki in 1972, see [5].

Theorem A. Let $f$ be holomorphic in $\mathbb{D}$ and such that $f(0)=f^{\prime}(0)-1=0$. If for all $z \in \mathbb{D}$ the inequality

$$
\left|\frac{z}{f(z)}-z\left(\frac{z}{f(z)}\right)^{\prime}-1\right|<1
$$

is valid, then $f$ is univalent in $\mathrm{D}$.

The present paper is devoted to the following simple generalization of this theorem for which we will provide an elementary proof.

Theorem 1. Let $f$ be meromorphic in $\mathbb{D}$ and such that $f(0)=f^{\prime}(0)-1=0$. If for all $z \in \mathbb{D}$ the inequality (1) is valid, then $f$ is univalent in $\mathbb{D}$.

Proof. The condition (1) implies immediately that $f$ has no zero in $\mathbb{D} \backslash\{0\}$. Further, there exists a function $\Omega$, holomorphic in $\mathbb{D}$ and satisfying the condition $|\Omega(z)|<1$ for $z \in \mathbb{D}$ such that

$$
\frac{z}{f(z)}-z\left(\frac{z}{f(z)}\right)^{\prime}-1=\Omega(z)
$$

2010 Mathematics Subject Classification. 30C45.

Key words and phrases. Meromorphic univalent functions, coefficient estimates .

File: PoWirths1²017'elem.tex, printed: 14-11-2018, 5.51.

The first author is currently at the ISI, Chennai Centre. 
The normalization of $f$ implies that $\Omega$ has a zero of multiplicity at least two at the origin. Therefore we may write $\Omega(z)=z^{2} \omega(z)$, where $\omega$ is analytic in $\mathbb{D}$ such that $|\omega(z)| \leq 1$ for $z \in \mathbb{D}$. The integration of the differential equation (2) delivers the representation

$$
\frac{z}{f(z)}=1+c z-z \int_{0}^{z} \omega(t) d t
$$

where $c$ is an arbitrary constant. This representation is known since long from the considerations of Theorem A, see for example the references in [6]. It is clear from the above that $f$ satisfies (1) if and only if it has the representation (3). The proof of the univalence is a one line proof now. Consider $z_{1}, z_{2} \in \mathbb{D}, z_{1} \neq z_{2}$ and

$$
\frac{f\left(z_{1}\right)-f\left(z_{2}\right)}{z_{1}-z_{2}}=\frac{1-\frac{z_{1} z_{2}}{z_{1}-z_{2}} \int_{z_{1}}^{z_{2}} \omega(t) d t}{\left(1+c z_{1}-z_{1} \int_{0}^{z_{1}} \omega(t) d t\right)\left(1+c z_{2}-z_{2} \int_{0}^{z_{2}} \omega(t) d t\right)} .
$$

Since

$$
\left|\int_{z_{1}}^{z_{2}} \omega(t) d t\right| \leq\left|z_{1}-z_{2}\right|
$$

we see that $f\left(z_{1}\right)-f\left(z_{2}\right) \neq 0$. This proves the univalence of $f$.

Remark 1. The difference between Theorem A and Theorem 1 is the fact that in Theorem 1, the function $f$ is allowed to have a pole in the unit disk. The proof of Theorem 1 shows that such pole is necessarily a simple pole.

We want to add the hint that similar meromorphic functions have been considered in $[2]$.

In what follows, we shall derive some analytic properties of the meromorphic functions satisfying (1). To that end we refine this condition, namely, we let $\lambda \in(0,1]$ and consider the condition

$$
\left|\frac{z}{f(z)}-z\left(\frac{z}{f(z)}\right)^{\prime}-1\right|<\lambda, z \in \mathbb{D} \text {. }
$$

For more details on the holomorphic functions satisfying such inequalities, we refer again to [6] and the references therein.

Theorem 2. (a) Let $f$ be meromorphic in $\mathbb{D}$ and such that $f(0)=f^{\prime}(0)-1=0$. If for all $z \in \mathbb{D}$ the inequality (1) is valid, then $f$ is continuous in $\overline{\mathbb{D}}$ with a possible exception at a zero of (3).

(b) Let $f$ be meromorphic in $\mathbb{D}$ and such that $f(0)=f^{\prime}(0)-1=0$. If for all $z \in \mathbb{D}$ the inequality (5) is valid for some $\lambda \in(0,1)$, then $f$ is univalent in $\overline{\mathrm{D}}$.

Proof. For the proof of (a) it is sufficient to recognize that (4) implies that

$$
A(z)=1+c z-z \int_{0}^{z} \omega(t) d t
$$


is uniformly continuous in $\mathbb{D}$. This implies the continuity of this function in $\overline{\mathbb{D}}$ and this in turn implies the continuity of $f$ in $\overline{\mathbb{D}}$ with the exception of the zeros of $A(z)$.

In the proof of (b) we use the fact that

$$
A_{\lambda}(z)=1+c z-\lambda z \int_{0}^{z} \omega(t) d t
$$

has a continuous extension to $\overline{\mathbb{D}}$ for $\lambda \in[0,1]$ which may be denoted by $A_{\lambda}(z)$, too. For $z_{1}, z_{2} \in \overline{\mathrm{D}}, z_{1} \neq z_{2}$, we get as in the proof of Theorem 1 that

$$
\left|\frac{z_{1} A_{\lambda}\left(z_{2}\right)-z_{2} A_{\lambda}\left(z_{1}\right)}{z_{1}-z_{2}}\right| \geq 1-\lambda\left|z_{1} z_{2}\right|>0
$$

for $\lambda \in[0,1)$. This implies

$$
\frac{f\left(z_{1}\right)-f\left(z_{2}\right)}{z_{1}-z_{2}} \neq 0
$$

and therefore the assertion of the theorem is proved.

Another typical problem in geometric function theory is the estimation of the moduli of the Taylor coefficients. Since in our case, the functions $f$ are holomorphic in a neighbourhood of the origin, we may ask for the upper bounds of the coefficients of the Taylor expansion in a disk around the origin. For the second coefficient we can give a satisfactory result using an elementary method from [6]. Also, compare with [2], too.

Theorem 3. Let $\lambda \in(0,1]$ and $f$ be meromorphic in $\mathbb{D}$ and such that $f(0)=$ $f^{\prime}(0)-1=0$. If for all $z \in \mathbb{D}$ the inequality (5) is valid and

$$
f(z)=z+\sum_{n=2}^{\infty} a_{n} z^{n}
$$

has no pole in the disk $\{z:|z|<p\}, p \in(0,1]$, then the inequality

$$
\left|a_{2}\right| \leq \frac{1+\lambda p^{2}}{p}
$$

is valid. The inequality is sharp and equality is attained if and only if

$$
f(z)=\frac{z}{\left(1-\frac{e^{i \theta} z}{p}\right)\left(1-\lambda p e^{i \theta} z\right)}, \quad \theta \in[0,2 \pi) .
$$

Proof. From the hypotheses above, we know that $f$ is of the form

$$
f(z)=\frac{z}{1+c z-\lambda z \int_{0}^{z} \omega(t) d t} .
$$

Obviously, $a_{2}=-c$. Now, we assume on the contrary that

$$
\left|a_{2}\right|>\frac{1+\lambda p^{2}}{p} \text {. }
$$

Then we can define

$$
\left|a_{2}\right|=\frac{1+\lambda p^{2}}{p r}
$$


for some $r \in(0,1)$. We consider the function

$$
F(z)=\frac{1}{a_{2}}\left(1-\lambda z \int_{0}^{z} \omega(t) d t\right)
$$

in the closed disk $\overline{\mathbb{D}}_{r p}:=\{z:|z| \leq p r\}$. Then we have the inequality

$$
|F(z)| \leq \frac{p r\left(1+\lambda p^{2} r^{2}\right)}{1+\lambda p^{2}}<p r
$$

which implies that $F$ maps this disk into itself. Secondly, for $z_{1}, z_{2}$ in the disk $\overline{\mathrm{D}}_{r p}$, we have

$$
\begin{aligned}
\left|F\left(z_{1}\right)-F\left(z_{2}\right)\right| & =\frac{\lambda r p}{1+\lambda p^{2}}\left|z_{1} \int_{0}^{z_{1}} \omega(t) d t-\left(z_{2}-z_{1}+z_{1}\right) \int_{0}^{z_{2}} \omega(t) d t\right| \\
& \leq \frac{\lambda r p}{1+\lambda p^{2}}\left(\left|z_{1}\right|\left|\int_{z_{2}}^{z_{1}} \omega(t) d t\right|+\left|z_{2}-z_{1}\right|\left|\int_{0}^{z_{2}} \omega(t) d t\right|\right) \\
& \leq \frac{\lambda r p\left(\left|z_{1}\right|+\left|z_{2}\right|\right)\left|z_{2}-z_{1}\right|}{1+\lambda p^{2}} \\
& \leq \frac{2 \lambda r^{2} p^{2}}{1+\lambda p^{2}}\left|z_{2}-z_{1}\right| .
\end{aligned}
$$

Since $2 \lambda r^{2} p^{2} /\left(1+\lambda p^{2}\right)<1$, the conditions of Banach's fixed point theorem are fulfilled and thus, the function $F$ has a fixed point in the closed disk $\{z:|z| \leq p r\}$. Therefore $f$ has a pole in the disk $\overline{\mathrm{D}}_{r p}$. This is a contradiction to the conditions of Theorem 3. It is easy to see that the functions (7) satisfy the conditions of the theorem and that for them equality in (6) is attained. To prove the rest of the assertion, we use a method from [6], where this has been shown in the case $p=1$. Therefore, we have to consider here only the cases $p<1$. Let us assume that there exists a function $\omega$ that is not of constant modulus one and is such that the function

$$
f(z)=\frac{z}{1-\frac{1+\lambda p^{2}}{p} e^{i \theta} z-\lambda z \int_{0}^{z} \omega(t) d t}
$$

satisfies the conditions of the theorem. According to the above assumption, there exists a number $c \in[0,1)$ such that for $|z| \leq p$ the inequality

$$
\left|\int_{0}^{z} \omega(t) d t\right| \leq c|z|
$$

is valid. We define

$$
r=\frac{1+\lambda c p^{2}}{1+\lambda p^{2}} \in(0,1)
$$

Now, we consider again the function (8) and we get by considerations similar to the above ones that $F$ is a contractive mapping of the closed disk $\{z:|z| \leq p r\}$ into itself. Hence, $F$ has a fixed point in this disk, and therefore there exists a pole of $f$ in the same disk. This again contradicts the above conditions. Now, it remains to prove that the only quadratic polynomials of the form

$$
1-\frac{1+\lambda p^{2}}{p} e^{i \theta} z-\lambda e^{i \varphi} z^{2}
$$


that have no zero in the disk $\{z:|z|<p\}$ are the polynomials

$$
1-\frac{1+\lambda p^{2}}{p} e^{i \theta} z+\lambda e^{2 i \theta} z^{2}
$$

This is a simple exercise of calculations and thus, we complete the proof.

Remark 2. In a neighbourhood of the origin, the functions (7) have the Taylor expansion

$$
f(z)=\sum_{n=1}^{\infty} \frac{1-\lambda^{n} p^{2 n}}{p^{n-1}\left(1-\lambda p^{2}\right)} e^{i n \theta} z^{n} .
$$

We conjecture that this delivers the upper bound

$$
\left|a_{n}\right| \leq \frac{1-\lambda^{n} p^{2 n}}{p^{n-1}\left(1-\lambda p^{2}\right)}
$$

for all functions $f$ satisfying the conditions of Theorem 3. In the case $\lambda=1$ and $p=1$, this is a special case of the famous Bieberbach conjecture that has been proved by de Branges in [3]. For $\lambda=1$ and arbitrary $p \in[0,1)$ the validity of the Bieberbach conjecture implies that the above conjecture is true. This has been proved by Jenkins in [4]. For $\lambda \in[0,1)$ and $p=1$ the truth of the above conjecture for $n=3$ and $n=4$ was shown in [7]. However the general conjecture remains open. We conclude the article by recalling that the above conjecture is a generalization of the following conjecture from [7].

Conjecture 1. Suppose that a holomorphic function $f$ in satisfies the condition (5) for some $0<\lambda \leq 1$. Then $\left|a_{n}\right| \leq \sum_{k=0}^{n-1} \lambda^{k}$ for $n \geq 2$.

\section{REFERENCES}

1. L. A. Aksentév, Sufficient conditions for univalence of regular functions (Russian), Izv. Vysš. Učebn. Zaved. Matematika 1958 (4)(1958), 3-7.

2. B. Bhowmik and F. Parveen, On a subclass of meromorphic univalent functions, Complex Var. Elliptic Equ. 62 (2017), 494-510.

3. L. de Branges, A proof of the Bieberbach conjecture, Acta Math. 20 (1985), 137-152.

4. J. A. Jenkins, On a conjecture of Goodman concerning meromorphic univalent functions, Michigan Math. J. 9 (1962), 25-27.

5. M. Nunokawa and S. Ozaki, The Schwarzian derivative and univalent functions, Proc. Amer. Math. Soc. 33 (1972), 392-394.

6. M. Obradović, S. Ponnusamy, and K.-J. Wirths, Geometric studies on the class $\mathcal{U}(\lambda)$, Bull. Malaysian Math. Sci. Soc. 39 (2016), 1259-1284.

7. M. Obradović, S. Ponnusamy, and K.-J. Wirths, Logarithmic coefficients and a coefficient conjecture for univalent functions, Monatshefte Math. (2017).

Online DOI 10.1007/s00605-017-1024-3

S. Ponnusamy, Department of Mathematics, Indian Institute of Technology Madras, Chennal-600 036, India.

E-mail address: samy@isichennai.res.in, samy@iitm.ac.in

K.-J. Wirths, Institut für Analysis und Algebra, TU Braunschweig, 38106 BraunSCHWEIG, GERMANY.

E-mail address: kjwirths@tu-bs.de 